\title{
ESTIMATION OF THE PARAMETERS OF LINEAR REGRESSION MODEL WITH AUTOCORRELATED ERROR TERMS WHICH ARE ALSO CORRELATED WITH THE REGRESSOR
}

(Received 4 May 2006; Revision Accepted 24 Septemher 2006)

\section{ABSTRACT}

This study used the Monte-Carlo method to investigate the performance of five estimators: Ordinary Least Squares (OLS), Cochrane Orcutt (CORC), Hildreth Lu (HILU), Maximum Likelihood (ML) and Maximum Likelihood Grid (MLGRID) in estimating the parameters of a single equation linear regression model in which the autoregressive independent variable is also correlated with the autoregressive error terms

The simulation results, under the finite sampling properties of Bias, Variance and Root Mean Squared Error, show that all estimators are adversely affected as autocorrelation coefficient $(\rho)$ is close to unity. In this regard, the estimators rank as follows in descending order of performance: OLS, MLGRID, ML, HILU and CORC

The estimators conform to the asymptotic properties of estimates considered. This is seen when the level of autocorrelation is mild (i.e. $\rho \leq 0.8$ ) at all significant levels. The estimators' rank in decreasing order in conformity with the observed asymptotic behaviour as follows: OLS, HILU, MLGRID, CORC and ML. Most of the criteria used in studying the relative performance of the five estimators have not exhibited any remarkable sensitivity to the number of replications. Increasing the number of replications has tended to confirm the stability of the study.

The results also suggest that OLS should be preferred when autocorrelation level is relatively mild $(\rho=0.4)$ and the regressor is significantly correlated (at least at $5 \%$ ) with the autocorrelated error terms.

This result helps in the choice of estimators in empirical work when the regressor and the error terms are not well behaved. it aiso allows correct inferences in linear models plagued by autocorrelated disturbances, which are also significantly correlated with the independent variable.

\section{KEVWORDS: Monte-Cario Experiment, GLS Estimators, Autocorrelated Error Terms, Linear Regression}

\section{INTRODUCTION}

$$
\begin{aligned}
& \text { regression model } \\
& Y Y=X \beta+U
\end{aligned}
$$

where $Y$ is a $(N \times 1)$ vector of observations, $X$ is a known ( $N \times$ K) non-stochastic design matrix of rank $K, \beta$ is a $(K \times 1)$ fixed vector of unknown parameters and $U$ is $a(N \times 1)$ vector of unobservable random variable with zero mean and finite covariance matrix. When the error terms follow a first order autoregressive (AR) process, we have

$$
\begin{gathered}
U_{1}=\rho U_{1-1}+\varepsilon_{1}, \quad|\rho|<1, \quad \varepsilon_{1} \rightarrow N I D\left(0, \sigma^{2}\right), \\
U_{0} \rightarrow N\left(0, \frac{\sigma^{2}}{1-\rho^{2}}\right)
\end{gathered}
$$

Assumptions in the classical normal linear regression model include that of lack of autocorrelation of the error terms and the zero covariances between the independent variable and the error terms. This paper examines the estimation of the parameters of the linear models when the above two assumptions are violated.

These violations are seen in widespread applications in operations research, like in queuing theory and econometrics, where the usual assumption of independent error terms may not be plausible in most cases. These violations are also observed when using time series data on a number of micro-economic units, for example, households; and also in service oriented channels, where the stochastic disturbance terms in part reflect variables which are not
- included explicitly in the model and which may change slowly over time ${ }^{17}$. Cochrane and Orcutt ${ }^{5}$ have shown that the error terms in most current formulations of economic relations are highly positively autocorrelated. Rao and Griliches ${ }^{20}$ have shown that there is much to gain and little to lose by considering alternatives to the independent error assumption of the classical linear regression model.

Many models with autocorrelated error terms have been discussed in the literature. These include the works of Anderson ${ }^{1}$, Cochran and Orcutt ${ }^{5}$, Durbin and Watson ${ }^{6,7.8}$, Rao and Griliches ${ }^{20}$, Beach and Mackinnon ${ }^{2}$, Kramer ${ }^{13,14}$, Busse. Jeske and Kramer ${ }^{4}$. Kramer and Hassler ${ }^{15}$, Nwabueze ${ }^{17}$, Kleiber $^{12}$, Kramer and Marmol ${ }^{16}$, Butte $^{3}$ and Olaomi ${ }^{18}$. Tests for detecting the presence of autocorrelation and alternative consistent methods of estimating linear models with autocorrelated disturbance terms have been proposed. However, in spite of these tests and estimation methods, a number of questions in connection with the estimation of the classical regression linear model with autocorrelated error terms and non-zero covariance between the independent variable and the error terms remained unanswered. These include the most appropriate estimation method in the above named specification of the independent variable, the effect of the degree of correlation of the disturbance term, the effect of the degree of correlation of independent variable and the error terms, the effect of replications and sample size and the sampling properties of the various estimation methods.

The answers to most of these questions would allow for correct inferences to be made in linear models plagued by the scenario depicted above.

The rest of this paper discusses the methodology, the model and the data generation procedure in section 2 , section 
3 presents the simulation results, and section 4 presents the discussions, while we conclude in section 5 .

\section{METHODOLOGY}

This study used the Monte-Carlo approach for the investigation due to the fact that real life observations on economic variables are in most cases plagued by one or several of the problems of non-spherical disturbances (a problem where the disturbance term $U$ in any period is correlated with any other value $U$ in the series, that is, serial correlation of the random variable $U$ ), measurement error and specification error. Also when the covariance between the independent variable and the error terms is non-zero, the problem is near intractable by analytical procedure.

The following four Generalised Least Squares (GLS) estimators (Cochrane and Orcutt (CORC), Hidreth and LU (HILU), Maximum Likelihood (ML) and Maximum Likelihood Grid (MLGRID)\} and Ordinary Least Squares (OLS) estimation methods, choosing in the light of the previous studies, are used. These estimators are equivalent with identical asymptotic properties ${ }^{15}$. But in small samples, such as in this study, Park and Mitchell ${ }^{19}$ have argued that those that use the $T$ transformation matrix (ML, MLGRID) are generally more efficient, in terms of estimation of the parameters of the model, than those that use $T^{*}$ transformation matrix (CORC, HILU)

The degree of autocorrelation affects the efficiency of the estimators ${ }^{17}$. Consequently, we investigated the sensitivity of the estimators to the degree of autocorrelation by varying rho $(\hat{\rho})$ from 0.4 , to 0.8 and 0.9 . We also found out the effect of the correlation of the independent variable and the error terms at significant level $(\alpha) 1 \%, 2 \%$ and $5 \%$ on the estimators. The effects of sample size $(N)$ and replication $(R)$ on the estimators were also investigated by varying the sample size from 20,40 to 60 each replicated 30,40 and 50 times Evaluation of the best estimator(s) was then done using the finite sampling properties of Minimum Bias (BIAS), Minimum Variance (VAR), and Minimum Root Mean Squared Error (RMSE).

\subsection{THE MODEL}

We assume a simple linear regression model:

$$
\begin{gathered}
Y_{1}=\beta_{0}+\beta_{1} X_{1}+U_{1} \\
U_{1}=\rho U_{t-1}+\varepsilon_{1}, \quad|\rho|<1, \\
X_{1}=\lambda X_{t-1}+V_{t}, \quad V_{1} \rightarrow N(0,1) \quad \lambda=0.8 \\
U_{1} \rightarrow N\left(\begin{array}{c}
\left.0 . \frac{\sigma^{2}}{1-\rho^{2}}\right), \quad X_{1} \rightarrow N\left(0, \frac{\sigma^{2}}{1-\lambda^{2}}\right) \\
t=1,2, \ldots N, \quad H=(1,1)
\end{array}\right.
\end{gathered}
$$

where $Y_{1}$ is the dependent variable and the first order autoregressive $X_{t}$ is the independent variable with $U_{t}$ also autoregressive of order one. $\varepsilon_{t}$ and $V_{t}$ are standard normal distributed. $\rho$ and $\lambda$ are stationarity parameters while the model parameters are assumed to be unity. Spitzer ${ }^{21}$. Nwabueze ${ }^{17}$ and Olaomi ${ }^{18}$ had used this independent variable' specification. It is chosen to allow for comparison of results.

\subsection{DATA GENERATION}

A total of 81 data sets spread over three sample sizes $(20,40$ and 60$)$ and three replication numbers $(30,40$, and 50$)$ were used in generating the data for this study. Using model (2), a value $U_{0}$ was generated by drawing a random value io. from $N(0.1)$ and dividing by $\sqrt{\left(1-\rho^{2}\right)}$. Successive values of $\varepsilon_{t}$ drawn from $N(0,1)$ were used to calculate $U_{t} . X_{t}$ was similarly generated. Correlation between $U_{1}$ and $X_{i}$ was then computed and its absolute value tested for significance say $1 \%$. If this value is significant, it is chosen, otherwise it is discarded. This procedure is repeated as many times as are necessary to obtain specified number of replications for a desired autocorrelation level, significance level and sample size. $Y_{t}$ is thus computed for the chosen $U_{1}$ and fixed $X_{t}$ using equation (2). The computations are made using the Excel package; different estimation methods are then applied to the data using the AR procedure of the TSP ${ }^{20}$ package

\section{SIMULATION RESULTS}

The finite sampling properties of estimators we used include the Bias (BIAS), Variance (VAR) and the Root Mean Squared Error (RMSE). Additionally, we calculated and displayed the Sum of Bias (SBIAS), Variances (SVAR) and the Root Mean Squared Error (SRMSE)

The results are summarized for SBIAS, SVAR and SRMSE as shown in Tables 1,2 , and 3 respectively for sample sizes 20 and 60 replicated 30,40 and 50 times each. (The result for sample size forty $(N=40)$ is omitted for page constraint, though, it is used in the explanation of results)

In the discussion of the results, attention is focused on comparison fof the following attributes of the estimates yielded by each of the five estimators:

(i) the sum of the bias of the two estimated parameters (SBIAS) with particular emphasis on sensitivity of these magnitudes to $\alpha, \rho, R$ and $N$, and

(ii) the sum of variances and the sum of the RMSE of the estimates also with emphasis to their sensitivity to $\alpha, p, R$ and $\mathrm{N}$ as in (i) above

Observing the trends followed by the estimates as i and $p$ varies, for each of Bias, Variance and the RMSE, it could be observed that all estimators are adversely affected as autocorrelation coefficient $(\rho)$ is close to unity when the regressor is significantly correlated with the error term. This is evidenced by the optimumf $(\rho, \alpha)$ combinations of $(0.4,0.01)$ as $\rho$ increases and $(0.4,0.05)$ as $\alpha$ decreases using both the Variance and the RMSE criteria while the Bias criterion give the optimum combination of $(0.8,0.05)$ with minimum occurring at $\rho=0.8$. There is absence of the combinations 10.9 . $0.01),(0.9,0.02),(0.9,0.05),(0.8,0.01)$ and $(0.8,0.02)$ which shows that the estumators perform less as $\rho \rightarrow 1$

The performance of the estimators rank as follows in descending order based on trends of Bias, Variance and RMSE: OLS, MLGRID, ML, HILU, and CORC.

We also investigated the asymptotic behaviour of the estimators in our experiment (i.e. as $\mathrm{N}$ increases). The five estimators rank as follows in decreasing order of conformity with the observed asymptotic behaviour of Bias, Variance and RMSE OLS. HILU, MLGRID, CORC, and ML.

In most Monte - Carlo studies, magnitudes such as bias, varriance and root mean squared error are not usually remarkably sensitive to the number of replications. We did not assume this a priori; hence the possible effects of different numbers of replication on these magnitudes are investigated.

The five estimators rank as follows in decreasing order of conformity with the observed replication effect of bias, variance and RMSE: OLS, CORC, ML, MLGRID, and HILU. The results of replication effect suggest that the behaviour of bias, variance and root mean squared error are remarkably less sensitive to replication numbers than sample sizes. Also, the optimum trend occurred at the same replication number 50 for bias, variance and RMSE, which suggests that the replications actually confirmed the stability of our results. 
Table 1: Sum of Absolute Bias for Estimators of $\beta$ for $N=20$ and $N=60$ (All replications)

\begin{tabular}{|c|c|c|c|c|c|c|c|c|c|c|}
\hline \multirow{2}{*}{$\begin{array}{l}\text { Significant } \\
\text { Level }\end{array}$} & \multirow[t]{2}{*}{ Estimator } & \multicolumn{3}{|l|}{$\rho=0.4$} & \multicolumn{3}{|l|}{$p=0.8$} & \multicolumn{3}{|l|}{$p=0.9$} \\
\hline & & $\begin{array}{l}N=20 \\
R=30\end{array}$ & $\begin{array}{l}N=20 \\
R=40\end{array}$ & $\begin{array}{l}N=20, \\
R=50\end{array}$ & $\begin{array}{l}N=20, \\
R=30\end{array}$ & $\begin{array}{l}N=20, \\
R=40\end{array}$ & $\begin{array}{l}N=20, \\
R=50\end{array}$ & $\begin{array}{l}N=20 \\
R=30\end{array}$ & $\begin{array}{l}N=20, \\
R=40\end{array}$ & $\begin{array}{l}N=20 . \\
R=50\end{array}$ \\
\hline \multirow{5}{*}{0.01} & OLS & 2.00672 & 2.01345 & 2.001397 & 2.019522 & 2.011611 & 1.979407 & 1.94924 & 1.976075 & 1.945679 \\
\hline & CORC & 2.009557 & 2.007646 & 1.98372 & 2.099689 & 2.085352 & 2.117686 & 2.007161 & 1.983825 & 1.969557 \\
\hline & HILU & 2.010673 & 2.009382 & 1.985357 & 1.971174 & 1.989489 & 2.05607 & 1.821298 & 1.797405 & 1.786586 \\
\hline & $\overline{\mathrm{ML}}$ & 2.002887 & 2.009204 & 1.99943 & 1.954783 & 1.949677 & 1.970245 & 1.965999 & 1.960984 & 1.990792 \\
\hline & MLGRID & 2.002931 & 2.009446 & 1.99984 & 1.946327 & 1.944887 & 1.939896 & 2.047327 & 2.04501 & 2.020209 \\
\hline \multirow{5}{*}{0.02} & OLS & 2.035417 & 2.027578 & 2.012331 & 0.058988 & 0.087823 & 0.053624 & 2.100742 & 2.102724 & 2.088479 \\
\hline & CORC & 2.071447 & 2.052589 & 2.05112 & 0.044664 & 0.072496 & 0.038231 & 2.164015 & 1.85121 & 1.850582 \\
\hline & HILU & 2.018253 & 2.023326 & 2.027572 & 0.069359 & 0.101422 & 0.061715 & 1.995361 & 1.938694 & $1 . \overline{980895}$ \\
\hline & $M L$ & 2.002364 & 1.985979 & 1.971339 & 0.053895 & 0.077344 & 0.050747 & 2.01348 & 1.975195 & 1.975273 \\
\hline & MLGRID & 1.989597 & 1.989994 & 1.992195 & 0.088088 & 0.102886 & 0.092551 & 2.059795 & 2.019704 & 1.998286 \\
\hline \multirow{5}{*}{0.05} & OLS & 1.999429 & 2.003305 & 2.000829 & 0.0561496 & 0.0334392 & 0.0664662 & 2049742 & 1.968029 & 1.982158 \\
\hline & $\overline{C O R C}$ & 1.979828 & $1.98337 \overline{6}$ & 1.994258 & 0.1011124 & 0.2348154 & 0.1686729 & 2.082196 & 2.082721 & 2.089437 \\
\hline & HILU & 2.003201 & 2.001 & 2.008555 & 0.088639 & 0.0888913 & 0.0529243 & 2.010138 & 2.032735 & 2.104394 \\
\hline & ML & 1.985907 & 1.993163 & 1.995076 & 0.087603 & 0.025485 & 0.0056 & 1966716 & 1.922908 & 1.947758 \\
\hline & MLGRID & $1 . \overline{987827}$ & 1.994408 & 1.996452 & 0.105352 & 0.0893324 & 0.0565083 & 1.980556 & 1.938704 & 1.966893 \\
\hline
\end{tabular}

\begin{tabular}{|c|c|c|c|c|c|c|c|c|c|c|}
\hline \multirow{2}{*}{$\begin{array}{l}\text { Significant } \\
\text { Level }\end{array}$} & \multirow[t]{2}{*}{ Estimator } & \multicolumn{3}{|l|}{$\rho=0.4$} & \multicolumn{3}{|l|}{$\rho=0.8$} & \multicolumn{3}{|l|}{$\rho=0.9$} \\
\hline & & $\begin{array}{l}N=60, \\
R=30\end{array}$ & $\begin{array}{l}N=60, \\
R=40\end{array}$ & $\begin{array}{l}N=60, \\
R=50\end{array}$ & $\begin{array}{l}\bar{N}=60, \\
R=30\end{array}$ & $\begin{array}{l}N=60, \\
R=40\end{array}$ & $\begin{array}{l}N=60, \\
R=50\end{array}$ & $\begin{array}{l}N=60, \\
R=30\end{array}$ & $\begin{array}{l}N=60 \\
R=40\end{array}$ & $\begin{array}{l}N=60, \\
R=50\end{array}$ \\
\hline \multirow{5}{*}{0.01} & OLS & 0.01296 & 0.01045 & 0.02452 & 0.020918 & 0.016128 & $0.00958 \overline{9}$ & 0.091205 & 0.072217 & 0.072096 \\
\hline & CORC & 0.05750 & 0.04417 & 0.04124 & 0.017076 & 0.020482 & 0.007208 & 0.037332 & 0.011274 & 0.024551 \\
\hline & HILU & 0.04027 & 0.03354 & 0.0327 & 0.017481 & 0.00989 & 0.0025244 & 0.09573 & 0.06727 & 0.06797 \\
\hline & $\overline{M L}$ & 0.06089 & $0.0454 \overline{4}$ & 0.04065 & 0.033145 & 0.015499 & 0.015663 & 0.045336 & 0.057495 & 0.086133 \\
\hline & MLGRID & 0.03861 & 0.02787 & 0.0273 & 0.029097 & 0.012525 & 0.031493 & 0.018391 & 0.043605 & 0.046388 \\
\hline \multirow{5}{*}{0.02} & OLS & 0.00211 & 0.001035 & 0.001623 & 0.026568 & 0.012778 & 0.008919 & 0.04865 & 0.06253 & 0.06218 \\
\hline & CORC & 0.016565 & 0.010162 & 0.011069 & 0.025391 & 0008719 & 0.014044 & 0.253943 & 0.217335 & 0.194355 \\
\hline & HILU & 0.015469 & 0.009561 & 0.010425 & 0.016038 & 0.012195 & 0.01728 & 0.070292 & 0.061384 & 0.024102 \\
\hline & $\mathrm{ML}$ & 0.008936 & 0.00884 & 0.005808 & 0017933 & 0.018277 & 0.02101 & 0.180547 & 0113496 & 0.040749 \\
\hline & MLGRID & 0.041458 & 0.026908 & 0.024178 & 0.02666 & 0.013897 & 0.0211114 & 0.153787 & 0.098974 & 0.046591 \\
\hline \multirow{5}{*}{0.05} & OLS & 0.002809 & 0.003382 & 0.003315 & 0.020296 & 0.026675 & 0.007642 & 0.064927 & 0.038539 & 0.034141 \\
\hline & CORC & 0.013529 & 0.01119 & 0.00572 & 0.05402 & 0.07636 & 0.0602 & $0 . \overline{124921}$ & 0.023225 & 0.026179 \\
\hline & HILU & 0.016459 & 0.014448 & 0.017928 & 0.0513 & 0.07616 & 0.06263 & 0.145867 & 0.062353 & 0.051439 \\
\hline & $M L$ & 0.015143 & 0.009646 & 0.010106 & 0.065352 & 0.085669 & 0.067492 & 0.180878 & 0.121945 & 0.068846 \\
\hline & MLGRID & 0.014375 & 0.008302 & 0.009429 & 0.044863 & 0.067861 & 0.072527 & 0.150453 & 0.093332 & 0.055344 \\
\hline
\end{tabular}


Table 2: Sum of Variances of Bias for Estimators of $\beta$ for $N=20$ and $N=60$ (All replications)

\begin{tabular}{|c|c|c|c|c|c|c|c|c|c|c|}
\hline \multirow{2}{*}{$\begin{array}{l}\text { Significant } \\
\text { Level }\end{array}$} & \multirow[t]{2}{*}{ Estimator } & \multicolumn{3}{|l|}{$\rho=0.4$} & \multicolumn{3}{|l|}{$\rho=0.8$} & \multicolumn{3}{|l|}{$\rho=0.9$} \\
\hline & & $\begin{array}{l}N=20, \\
R=30\end{array}$ & $\begin{array}{l}N=20, \\
R=40\end{array}$ & $\begin{array}{l}N=20, \\
R=50\end{array}$ & $\begin{array}{l}N=20, \\
R=30\end{array}$ & $\begin{array}{l}N=20, \\
R=40\end{array}$ & $\begin{array}{l}\mathrm{N}=20, \\
\mathrm{R}=50\end{array}$ & $\begin{array}{l}N=20 . \\
R=30\end{array}$ & $\begin{array}{l}N=20, \\
R=40\end{array}$ & $\begin{array}{l}N=20 \\
R=50\end{array}$ \\
\hline \multirow{5}{*}{0.01} & OLS & 0.2917708 & 0.2770682 & 0.2600066 & 0.7306049 & 0.6730976 & 0.6360087 & 1.2950126 & 1.2009676 & 1.1410226 \\
\hline & CORC & 0.2831761 & 0.2656922 & 0.2505263 & 0.8270936 & 0.6898761 & 0.6527618 & 1.0194169 & 0.8708283 & 0.7657319 \\
\hline & HILU & 0.2833661 & 0.2660475 & 0.2510355 & 0.6192805 & 0.5347448 & 0.5481383 & 0.9735029 & 0.892521 & 0.8196442 \\
\hline & $\mathrm{ML}$ & 0.2818188 & 0.2630449 & 0.2469734 & 0.4580663 & 0.4167675 & 0.4139603 & 1.2254239 & 1.1767008 & 1.1163396 \\
\hline & MLGRID & 0.2815797 & 0.2628911 & 0.2466277 & 0.4414921 & $0 . \overline{4043617}$ & 0.4023914 & 1.1775677 & 1.1154045 & 1.0340933 \\
\hline \multirow{5}{*}{0.02} & OLS & 0.1393636 & 0.1359369 & 0.1344286 & 0.2058534 & $0 . \overline{2172545}$ & 0.2089693 & 0.5903161 & 0.6773591 & $0 . \overline{6634187}$ \\
\hline & CORC & $0 . \overline{1342936}$ & 0.136505 & 0.1354404 & 0.1778809 & 0.1591779 & 0.1500322 & 2.192935 & 3.7476047 & 3.1649482 \\
\hline & HILU & 0.1636967 & 0.1523624 & 0.1479018 & 0.2136975 & 0.1905953 & 0.1762796 & 0.3998344 & 0.3735938 & 0.3769719 \\
\hline & $\overline{M L}$ & 0.1369034 & 0.154189 & 0.1673877 & 0.231402 & 0.2150166 & 0.200496 & 0.4249026 & 0.4181026 & 0.4358225 \\
\hline & MLGRID & 0.1528453 & 0.1425759 & 0.1396 & 0.2543282 & 0.2314688 & 0.2223818 & 0.4372153 & 0.4426205 & 0.4567298 \\
\hline \multirow{5}{*}{0.05} & OLS & 0.1202272 & 0.1175814 & 0.1155771 & 0.2482037 & 0.2294696 & 0.2231072 & 0.5956265 & 0.6403861 & 0.5958598 \\
\hline & CORC & 0.0957375 & 0.0970671 & 0.097874 & 0.5256693 & 1.4968368 & 1.2303207 & 1.5197523 & 1.5531538 & 1.267398 \\
\hline & HILU & $0 . \overline{0960903}$ & 0.097347 & 0.098252 & 0.4367708 & 0.4688644 & 0.3981557 & 0.2512618 & 0.2622081 & 0.3752807 \\
\hline & $M L$ & 0.0955999 & 0.0960047 & 0.0925097 & $0.21887 \overline{95}$ & 0.2088446 & 0.1958921 & $0 . \overline{42803}$ & $0.4 \overline{470161}$ & 0.4230614 \\
\hline & MLGRID & 0.0946084 & 0.0949742 & 0.0915859 & 0.2556906 & $0 . \overline{2419552}$ & 0.2236712 & 0.4201651 & 0.4348797 & $0 . \overline{4139272}$ \\
\hline
\end{tabular}

\begin{tabular}{|c|c|c|c|c|c|c|c|c|c|c|}
\hline \multirow{2}{*}{$\begin{array}{l}\text { Significant } \\
\text { Level }\end{array}$} & \multirow[t]{2}{*}{ Estimator } & \multicolumn{3}{|l|}{$\rho=0.4$} & \multicolumn{3}{|l|}{$\rho=0.8$} & \multicolumn{3}{|l|}{$p=0.9$} \\
\hline & & $\begin{array}{l}\mathrm{N}=60, \\
\mathrm{R}=30\end{array}$ & $\begin{array}{l}\mathrm{N}=60, \\
\mathrm{R}=40\end{array}$ & $\begin{array}{l}N=60, \\
R=50\end{array}$ & $\begin{array}{l}N=60, \\
R=30\end{array}$ & $\begin{array}{l}N=60 \\
R=40\end{array}$ & $\begin{array}{l}N=60, \\
R=50\end{array}$ & $\begin{array}{l}N=60 \\
R=30\end{array}$ & $\begin{array}{l}N=60, \\
R=40\end{array}$ & $\begin{array}{l}N=60, \\
R=50\end{array}$ \\
\hline \multirow{5}{*}{0.01} & OLS & 0.176368 & 0.158899 & 0.1642362 & 0.517286 & 0.459528 & 0.4374466 & 1.394178 & 1.191885 & 1.1364449 \\
\hline & CORC & $0 . \overline{1057766}$ & 0.095094 & 0.0916026 & 0.072525 & 0.068183 & 0.0692881 & 0.220702 & 0.191118 & 0.1819406 \\
\hline & HILU & 0.107703 & 0.09647 & 0.0926124 & 0.081027 & 0.074721 & 0.0765582 & 0.184252 & 0.150104 & 0.1455753 \\
\hline & $\overline{M L}$ & 0.122844 & 0.105467 & 0.9812212 & 0.073347 & 0.06987 & 0.0683459 & 0.182376 & 0.153864 & 0.1607063 \\
\hline & MLGR & 0.102877 & 0.090692 & 0.0865706 & 0.074704 & 0.071007 & 0.0868902 & 0.1312 & 0.119674 & 0.1122606 \\
\hline \multirow{5}{*}{0.02} & OLS & 0.079563 & 0.074993 & 0.0736084 & 0.176403 & 0.173018 & 0.1666772 & 0.45346 & 0.402187 & 0.391828 \\
\hline & CORC & 0.055579 & 0.053211 & 0.0526387 & 0.044403 & 0.039953 & 0.0366614 & 1.499516 & 1.106077 & 0.9052559 \\
\hline & HILU & 0.054644 & 0.052807 & 0.0521946 & 0.047191 & 0.041455 & 0.0374849 & 0.080429 & 0.105857 & 0.1002894 \\
\hline & $\mathrm{ML}$ & 0.078449 & 0.068465 & 0.0643163 & 0.041674 & 0.040717 & 563 & 0.121253 & 0.132635 & 0.1546279 \\
\hline & MLGRID & 0.08 & 0.071261 & 0.0 & 0.046266 & 0.046218 & 247 & 0.126422 & 0.122912 & 0.1265501 \\
\hline \multirow{5}{*}{0.05} & OLS & 0.066635 & 0.064482 & 0.0636957 & 0.137192 & 2738 & $6 \overline{65}$ & 0.337234 & 0.353237 & 0.3362023 \\
\hline & CORC & 0.050026 & 0.065585 & 0.0632818 & 0.038182 & 0.033103 & $0.03 \overline{27175}$ & 0.075943 & 0.19537 & 0.1909612 \\
\hline & HILU & 0.050365 & 0.044886 & 0.0468954 & 0.039983 & 0.034771 & 0.033373 & 0.058576 & 0.094843 & 0.0935037 \\
\hline & $\overline{M L}$ & 0.043962 & 0.039757 & 0.041602 & 0.048031 & 0.040245 & 0.0377033 & 0.183619 & 0.171501 & 0.1967271 \\
\hline & MLGRID & 0.04497 & 0.040363 & 0.0421535 & 0.037801 & 0.032457 & 0.0428181 & 0.13729 & 0.140487 & 0.1479234 \\
\hline
\end{tabular}

Table 3: Sum of RMSE of Bias for Estimators of $\beta$ for $N=20$ and $N=60$ (All replications)

\begin{tabular}{|c|c|c|c|c|c|c|c|c|c|c|}
\hline \multirow{2}{*}{$\begin{array}{l}\text { Significant } \\
\text { Level }\end{array}$} & \multirow[t]{2}{*}{ Estimator } & \multicolumn{3}{|l|}{$\rho=0.4$} & \multicolumn{3}{|l|}{$\bar{\rho}=0.8$} & \multicolumn{3}{|l|}{$\rho=0.9$} \\
\hline & & $\begin{array}{l}N=20, \\
R=30\end{array}$ & $\begin{array}{l}N=20, \\
R=40\end{array}$ & $\begin{array}{l}N=20, \\
R=50\end{array}$ & $\begin{array}{l}N=20, \\
R=30\end{array}$ & $\begin{array}{l}N=20, \\
R=40\end{array}$ & $\begin{array}{l}N=20, \\
R=50\end{array}$ & $\begin{array}{l}N=20, \\
R=30\end{array}$ & $\begin{array}{l}\mathrm{N}=20, \\
\mathrm{R}=40\end{array}$ & $\begin{array}{l}N=20 \\
R=50\end{array}$ \\
\hline \multirow{5}{*}{0.01} & OLS & 2.1463831 & 2.1457388 & 2.126307 & 2.3499986 & 2.3172336 & 2.2729925 & 2.5031266 & 2.490157 & 2.4173733 \\
\hline & CORC & 2.1452809 & 2.1355531 & 2.105471 & $2.46 \overline{2485}$ & 2.3940988 & 2.4069304 & 2.4647014 & 2.3835384 & 2.3268551 \\
\hline & HILU & 2.1463955 & 2.1373491 & 2.1072543 & 2.2629714 & 2.2424531 & 23075708 & 2.3034195 & 2.2433428 & 2.2028399 \\
\hline & $M L$ & 2.1381025 & 2.1356164 & 2.1185449 & 2.1743471 & 2.1504862 & 21672798 & 2.5181316 & 2.4926103 & 2.4889467 \\
\hline & MLGRID & 2.1379813 & 2.1357456 & 2.118759 & 2.1569248 & 2.138691 & 2.1317296 & 2.5544234 & 2.5272226 & 2.4754435 \\
\hline \multirow{5}{*}{0.02} & OLS & 2.1030006 & 2.0937159 & 2.0779932 & 0.6282435 & 0.644284 & 0.6307173 & 2.361669 & 2.3984561 & 2.3807055 \\
\hline & CORC & 2.1354608 & 2.1180829 & 2.1161594 & 0.5984365 & 0.5693603 & 0.5492479 & 2.8817568 & 3.1000047 & 2.9631635 \\
\hline & HILU & 2.0978876 & 2.0974097 & 2.0993738 & 0.6575598 & 0.6256405 & 0.5969537 & 21823687 & 2.1198413 & 2.1585131 \\
\hline & $\mathrm{ML}$ & 2.0692482 & 2.0619373 & 2.0543535 & 0.6794952 & 0.6574901 & 0.6328284 & 2.2053012 & 2.1680719 & 2.1764571 \\
\hline & MLGRID & 2.0646949 & 2.0 & 954 & 0.7132915 & 0.68 & 0.6690777 & 2.2486543 & 2.2150724 & 2.203685 \\
\hline \multirow{5}{*}{0.05} & OLS & 2.0584924 & 2.060925 & 2.0575592 & 0.6950253 & 0.666721 & 0.6563293 & 2.3185126 & 2.268946 & 2.2600278 \\
\hline & CORC & 2.0276345 & 2.0317627 & 2.0427828 & 0.9593283 & 1.4901532 & 1.3663103 & 2.6426014 & 2.6621549 & 2.575431 \\
\hline & HILU & 2.0504206 & 2.0489425 & 2.0567887 & 0.8954525 & 0.9140499 & 0.8510265 & 2.1306611 & 2.1568205 & 2.2757302 \\
\hline & $\overline{M L}$ & 2.0333824 & 2.040713 & 2.0408009 & 0.6642964 & 0.6435349 & 0.625306 & 2.1660587 & 2.1406656 & 2.1520211 \\
\hline & $\overline{M L G R I D}$ & 2.0348269 & 2.0414671 & 2.041734 & 0.7247571 & 0.6995756 & $0 . \overline{670853}$ & 2.1751938 & 2.1497901 & 2.163981 \\
\hline
\end{tabular}




\begin{tabular}{|c|c|c|c|c|c|c|c|c|c|c|}
\hline \multirow{2}{*}{$\begin{array}{l}\text { Significant } \\
\text { Level }\end{array}$} & \multirow[t]{2}{*}{ Estimator } & \multicolumn{3}{|l|}{$\rho=0.4$} & \multicolumn{3}{|l|}{$p=0.8$} & \multicolumn{3}{|l|}{$p=0.0$} \\
\hline & & $\begin{array}{l}N=60, \\
R=30\end{array}$ & $\begin{array}{l}N=60, \\
R=40\end{array}$ & $\begin{array}{l}N=60, \\
R=50\end{array}$ & $\begin{array}{l}N=60, \\
R=30\end{array}$ & $\begin{array}{l}N=60 . \\
R=40\end{array}$ & $\begin{array}{l}N=60, \\
R=50\end{array}$ & $\begin{array}{l}N=60, \\
R=30\end{array}$ & $\begin{array}{l}N=60, \\
R=40\end{array}$ & $\begin{array}{l}N=60, \\
R=50\end{array}$ \\
\hline \multirow{5}{*}{0.01} & OLS & 0.432837 & 0.417545 & 0.510646 & 0.798578 & 0.771886 & 0.758931 & 1.478458 & 1.300302 & 1.361902 \\
\hline & CORC & 0.364423 & 0.348959 & 0.343160 & 0.358727 & 0.348169 & 0.351424 & 0.608653 & 0.575312 & 0.563226 \\
\hline & HILU & 0.365166 & 0.350318 & 0.344064 & $0 \times 388868$ & 0.374297 & 0.379615 & 0.596516 & 0.54424 & 0.538652 \\
\hline & $\overline{M L}$ & 0.371134 & 0.347982 & 0.337455 & 0.36433 & 0.356154 & 0.351744 & 0.566072 & 0.632515 & 0.544283 \\
\hline & MLGRID & 0.338586 & 0.322367 & 0.315461 & 0.369397 & 0.36049 & 0.392958 & 0.492167 & 0.470582 & 0.460345 \\
\hline \multirow{5}{*}{0.02} & OLS & 0.289108 & 0.267262 & 0.287064 & 0.499281 & 0,499618 & $0: 487889$ & 0.924996 & 0.88136 & 0.867076 \\
\hline & CORC & 0.262365 & $0 . \overline{261914}$ & 0.263569 & 0.294048 & 0.282924 & 0.271163 & 1.347603 & 1.105005 & 1.09500 \\
\hline & HILU & 0.260199 & 0.260856 & 0.262112 & 0.302649 & 0.288182 & 0.274514 & 0.38191 & 0.451763 & 0.430026 \\
\hline & $\overline{M L}$ & 0.292932 & 0.278016 & 0.26989 & 0.284472 & 0.28511 & 0.277913 & 0.481006 & 0.482905 & 0.534030 \\
\hline & MLGRID & 0.301111 & 0.284509 & 0.275033 & 0.299567 & 0.301843 & 0.298131 & 0.47942 & 0.467303 & 0.468303 \\
\hline \multirow{5}{*}{0.05} & OLS & 0.274168 & 0.273427 & 0.270894 & 0.446598 & 0.442921 & 0.439856 & 0.78876 & 0.834229 & 0.8128 \\
\hline & CORC & 0.25861 & 0.29465 & 0.289281 & 0.281603 & 0.271409 & 0.265086 & 0.378365 & 0.336427 & 0.538272 \\
\hline & HाLU & 0.2604 & 0.25085 & 0.256657 & 0.286684 & 0.277325 & 0.268568 & 0.358037 & 0.405429 & 0.408226 \\
\hline & $\mathrm{ML}$ & 0.237609 & 0.227659 & 0.229851 & 0.313517 & 0.297066 & 0.283936 & 0.555445 & 0.531554 & 0.654473 \\
\hline & MLGRिID & 0.245459 & 0.233951 & 0.235308 & 0.275384 & 0.26423 & 0.296059 & 0.475221 & 0.476101 & 0.405020 \\
\hline
\end{tabular}

4.0

\section{DISCUSSION OF RESULT}

The simulation results, under all the finite propenties considered show that all estimators are adversely affected as autocorrelation coefficient $(\rho)$ is close to unity when the regressor is significantly correlated with the autocorrelated error terms. This conforms to literature when there is no correlation between the regressor and the error terms. (See Green ${ }^{9}$, Verbeck ${ }^{23}$, Johnston and DiNardo ${ }^{11}$, Nwabueze ${ }^{i 7}$ ). In this regard, the estimators rank as follows in descending order: OLS, MLGRID, ML, HILU and CORC.

The results suggest that OLS should be preferred when autocorrelation revel is relatively mild $(\rho=0.4)$ and the regressor is significantly correlated (at least at $5 \%$ ) with the autocorrelated error term. This seems plausible because the corrective measures incorporated into the GLS (CORC, HILU, ML, MLGRID) estimators make use of the 'badly behaved regreseor' (negressor correlated with error terms) and these may adversely affect the performance of these estimators. The OLS estimator does not correct for autocorrelation and is therefore, not affected by this problem.

We found that the estimators conform to the asymptotic properties of estimates considered. This is seen when the level of autocorrelation is mild (i.e. $\rho \leq 0.8$ ) at all significant levels. The estimators' rank in decreasing order of conformity with the observed asymptotic behaviour is as follows: OLS, HILU, MLGRID, CORC and ML. This ranking is contrary to that of Nwabueze ${ }^{17}$ when there is independence between the regressor and the error terms.

Most of the criteria used in studying the relative perfor ance of the five estimators have not exhibited any remarkable sensitivity to the number of replications. Increasing the numbigr of replications has tended to confirm the stability of the study.

We also note that ML and MLGRID have very similar behavioural pattern, the same for CORC and HILU as observed in the finite sampling properties of Bias, Variance and the RMSE.

\subsection{CONCLUSION}

We have shown that when there is correlation between the regressor and the error terms in a classical simple linear regression estimation problem, OLS estimation method should be used based on the finite sampling criteria used in this experiment. It is also shown that all the estimators are still asymptotically behaved based on the criteria used, with OLS estimation method performing best.

\section{REFERENCES}

Anderson, R.L., 1948. "Distribution of the Serfal Correlation Coefficient". Annals of Mathematical Statistics., 13, 113.

Beach, C. M. and Mackinnon, J. S., 1978. "A Maximum Likelhood Procedure for Regresion with Autocorrelated Ertors". Economotrica, 46, (1): 51-57.

Butte Gotu, 2002. "The Equality of OLS and GLS Estimalore in the Linear Regression Model when the Disturbancase are Spatially Correlated". Statistical Papers. 42 (2): 253-263.

Busse, R., Jeske, R. and Kramer, W., 1994. "Efficiency of Least-Squares Estimation of Polynomial Trend when Residuals are Autocorrelated". Economica Leumere 45, 287-271.

Cochrane, D. and Orcutt, Q.H., 1949. "Application of Lesat Square Regression to Relationships Containing Autocorrelated Error Terms". Journal of the Americen Statistical Association, 44, 32-61.

Durbin, J. and Watson, G.S.; 1950. "Testing for Seriw Correlation in Least Squares Regreacion 1.". Biometrika, 37, 408-428.

Durbin, J. and Watson, G.J., 1951 "Teating for Seris Correlation in Least Squares Regresaion IF, Biometrika, 38, 159-178.

Durbin, J. and Watson, G.S. 1971 "Test for Serial Correlation in Least Squares Regression III", Biometrka, 58, 1. 42 .

Green, W. H., 2000. Econometric Analysis, Prentice-Hall, Upper Saddle River, New Jersey, $4^{\text {th }}$ Edition.

Hildreth, C. and LU, J.Y., 1960. "Demand Relationships with Autocorrelated Disturbances". Michigan State University. Agricultural Expt. Statn. Bullotin 276, East Lansing, Michigan

Johnston, J. and DiNardo, J., 1997. Econometric Methods. McGraw Hill, New York. $4^{\text {th }}$ Edition.

Kleiber, Christian, 2001. "Finite Sample Efficiency" of OLS in Linear Regression Models with Long-Momory Disturbances". Economic Letters 72, 131-136. 
Kramer, W., 1998. "Asymptotic Equivalence of Ordinary Least Squares and Generalized Least Squares with Trending Regressors and Stationary Autoregressive Disturbances". In Galata/Kutchenhoff (eds.): Econometrics in Theory and Practice (Festschrift for Hans Schneewei $\beta$ ), 137-142.

Kramer, W., 1980. "Finite Sample Efficiency of Ordinary Least Squares in the Linear Regression Model with Autocorrelated Errors". Journal of the American Statistical Association, 75, 1065-1067.

Kramer, W. and Hassler, U., 1998. "Limiting Efficiency of OLS Vs. GLS when Regressors are Fractionally Integrated". Economic Letters 60, 285-290.

Kramer, W. and Marmol F., 2002. "OLS-based Asymptotic Inference in Linear Regression Models with Trending Regressors and AR(P) Disturbances". Communications in Statistics - Theory and Methods, $31,2,2002,261-270$

Nwabueze, J. C., 2000. Estimation of Parameters of Linear Regression Models with Autocorrelated Error terms. Unpublished Ph.D. Thesis. University of Ibadan, Nigerià.
Olaomi. J. O., 2004. Estimation of Parameters of Linear Regression Models with Autocorrelated Error terms which are also correlated with the regressor. Unpublished Ph.D. Thesis. University of Ibadan, Nigeria.

Park, R.E. and Mitchell, B.M., 1980. "Estimating the Autocorrelated Error Model with Trended Data". Journal of Econometrics, 13, 185-201.

Rao, P. and Griliches, Z., 1969. "Small Sample Properties of Several Two-stage Regression Methods in the Context of Autocorrelated Errors". Journal of the American Statistical Association, 64, 251-272.

Spitzer, J.J., 1979. "Small Sample Properties of Nonlinear Least Squares and Maximum Likelihood Estimators in the Context of Autocorrelated Errors". Joumal of the American Statistical Association, 74, 126-138.

TSP, 2005. Users Guide and Reference Manual. Time Series Processor. New York.

Verbeck, M., 2000. A guide to Modern Econometric. Wiley, Chichester. 